\title{
Methylenetetrahydrofolate reductase gene C677T and A1298C polymorphisms and susceptibility to recurrent pregnancy loss
}

\author{
DOMENICO DELL'EDERA, ANTONELLA L'EPISCOPIA, FRANCESCA SIMONE, MARIA GIOVANNA LUPO, \\ ANNUNZIATA ANNA EPIFANIA and ARIANNA ALLEGRETTI
}

\author{
Unit of Cytogenetic and Molecular Genetics, 'Madonna delle Grazie’ Hospital, I-75100 Matera, Italy
}

Received October 2, 2017; Accepted November 24, 2017

DOI: $10.3892 /$ br.2018.1039

\begin{abstract}
Several studies have investigated the link between two different polymorphisms (C677T and A1298T) of the gene encoding methylenetetrahydrofolate reductase (MTHFR) and the risk of recurrent pregnancy loss (RPL); however, the results remain controversial. This study aimed to provide greater insight into this debated topic. In the current study, two groups of pregnant women (group A: RPL women; group B: non-RPL women), each of which were subdivided further into two subgroups based on their gestational age, were screened for C677T and A1298T variants of the MTHFR gene. The resulting data were analyzed using receiver operating characteristic (ROC) curve and $\mathrm{Z}$ test methods to compare the two groups. These ROC curve and $\mathrm{Z}$ test analyses indicated that there were no differences between the groups regarding C677T and A1298T expression. RPL is primarily caused by mutations in prothrombin or factor V Leiden genes. However, a low percentage of RPL cannot be attributed to these mutations. In the last five years, research has focused on the MTHFR gene, the two major variants of which (C677T and A1298T) have been associated with an increased risk of cardiovascular diseases (thrombotic events) in homozygous individuals. In addition, these mutations may be related to an increased rate of neural tube defects in fetuses. While a link between MTHFR mutation and RPL may be expected based on previous findings, the present study indicated the absence of an association between the polymorphisms of the MTHFR gene and RPL risk.
\end{abstract}

\section{Introduction}

In the last five years, several studies have evaluated whether a correlation between the C677T and A1298C polymorphisms of

Correspondence to: Dr Domenico Dell'Edera, Unit of Cytogenetic and Molecular Genetics, 'Madonna delle Grazie' Hospital, Contrada Cattedra Ambulante, I-75100 Matera, Italy

E-mail: domenicodelledera68@gmail.com

Key words: methilenthetrahydrofolate reductase, homocysteine, folic acid, C677T and A1298C polymorphisms, recurrent pregnancy loss the methylenetetrahydrofolate reductase (MTHFR) gene and a higher risk of recurrent pregnant loss (RPL) exists $(1,2)$. The C677T and A1298C variants of the MTHFR gene, in certain conditions, may lead to an increase in plasma homocysteine (Hcy) and homocysteineemia, which can cause endothelial damage in blood vessels (3). This may increase thromboembolic risk, which in pregnant women can lead to an obstruction of the placental vessels resulting in recurrent abortions. Thus, hyperhomocysteinemia is considered a risk factor for recurrent pregnancy loss (RPL), and patients with RPL may exhibit hyperhomocysteinemia; therefore, as part of routine check-up for RPL, serum homocysteine should be measured. Once diagnosed, treatment of hyperhomocysteinemia with folic acid and vitamin B12 is able to markedly decrease homocysteine levels (3).

Homocysteine (Hcy) is a non-essential, sulfur-containing, non-proteinogenic amino acid. It is an intermediate metabolite in the synthesis of methionine (remethylation reaction) and in the synthesis of cysteine (transulfuration reaction) (3). The enzymes involved in these metabolic pathways are: i) MTHFR, the coenzyme for which is vitamin B9, also known as folic acid (FA)/ folate or pteroyl-L-glutamate; ii) methionine synthetase (MS), which uses vitamin B12 (cobalamin) as a cofactor; and iii) cystathionin- $\beta$-synthetase (CBS), with vitamin B6 as its coenzyme (3).

The active form of FA in an organism is derived from a reduction reaction of FA into tethrahydrofolic acid (tethrahydrofolate, THF), with the latter being the true coenzyme of MTHFR. MTHFR and MS are involved in the recycling of Hcy into methionine (remethylation reactions), whereby Hcy acquires a methyl group from 5-MTHF to form the methionine (3). A mild or moderate form of hyperhomocysteinemia can be caused by folate deficiency, a direct or indirect lack of 5-methilentethrahydrofolate, a functional defect in MTHFR, MS, CBS orB9, or deficiencies in B12 and B6 vitamins (3).

MTHFR catalyzes irreversible conversion of 5,10-methylentethrahydrofolate to 5-methiltethrahydrofolate (CH3-THF). The normal activity of MTHFR aids to maintain folate and methionine in the bloodstream at constant levels, preventing Hcy accumulation (3). Polymorphisms in the gene encoding MTHFR may lower its enzymatic activity. The variant C677T leads to a substitution of a cytosine into a thymine at position 677 within exon 4 of the MTHFR gene. This genetic variant leads to an amino acidic substitution in position 222 
at the protein level (p.Ala $>\mathrm{Val})$ (3). Individuals carrying the MTHFRA222V variant in heterozygous form (genotype Ala/Val) can exhibit a $35 \%$ reduction in enzymatic activity relative to the basal activity level of non-carriers, while homozygotes for the variant (genotype Val/Val) can exhibit a $30 \%$ reduction in enzymatic activity relative to non-carriers (4). An additional MTHFR polymorphism associated with a reduction in enzyme activity is the substitution of an adenine with a cytosine in position 1,298 (A1298C) (5,6). This variation leads to the substitution of a glutamate with an alanine in position 429 at the protein level (p.429Glu>Ala) (7). It has been demonstrated that the C677T and A1298C polymorphisms in heterozygous form, along with a folate deficiency, lead to increased levels of plasmatic Hcy (7).

Patients carrying the C677T polymorphism of MTHFR in homozygous form (TT), leading to a thermolabile MTHFR variant, exhibit decreased formation of $\mathrm{CH} 3-\mathrm{THF}$ under conditions of folate deficiency: This leads to lower production of S-adenosylmethionine and subsequently lower availability of methyl groups (-CH3) required for methylation events, including the methylation of DNA $(8,9)$. Additionally, the lower availability of CH3-THF results in reduced remethylation of Hcy, followed by an increase in plasmatic Hcy (hyperhomocysteinemia) (8). Conversely, wild-type individuals for the C677T genotype (CC) are not influenced by folate deficiency, and thus CH3-THF synthesis for the methylation reaction and for the conversion of Hcy in methionine is preserved. Regarding these processes, the C677T and A1298C genotypes should not affect the availability of CH3-THF under an adequate supply of folate (9). However, current methods are unable to delineate a direct correlation between the aforementioned variants of the MTHFR gene and thromboembolic risk, and in turn, risk of RPL in pregnant women. The aim of the present study was to investigate the putative association between the C677T and A1298C variants and RPL risk.

\section{Subjects and methods}

Subjects. A total of 767 pregnant women (mean age, $30 \pm 1.2$ years) who visited the Unit of Cytogenetic and Molecular Genetics, 'Madonna delle Grazie' Hospital (Matera, Italy) from January 2010 to July 2016 to undergo molecular tests for the detection of the C677T and A1298C variants of the MTHFR gene were enrolled. The pregnant women were divided into two groups (group A and group B) based on miscarriage history. The present study protocol was approved by the Ethics Committee of Basilicata University (Potenza, Italy) and all participants provided written informed consent.

Pregnant women belonging to group A $(n=380)$ had positive anamnesis of at least two miscarriage events. These women exhibited negative responses to antithrombin deficiency, anticoagulant $\mathrm{C}$ protein and protein $\mathrm{S}$ tests, exhibited no increased resistance to activated C protein, had neither the A20210G mutation in prothrombin gene nor the G1691A mutation in Leiden factor $\mathrm{V}$ gene (p.Arg506Gln). They also had a negative response to antiphospholipid antibodies. The pregnant women belonging to group $B(n=387)$ were experiencing their second or third pregnancy and had negative anamnesis for miscarriage, as well as negative results in the aforementioned clinical tests. All enrolled patients and their partners carried a normal karyotype.

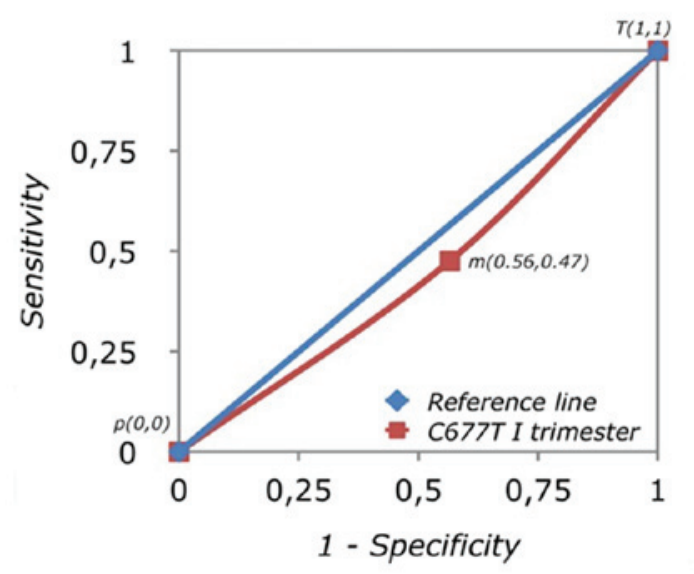

Figure 1. Receiver operating characteristic curve for C677T genotype in the first trimester of pregnancy. The point $\mathrm{m}$ with coordinates $(0.56,0.47)$ together with the coordinate points $(0,0),(1,1)$ and $(1,0)$ defines a quadrilateral. The latter area was given by: $\mathrm{A}=1 / 2$ specificity+ $1 / 2$ sensitivity; $\mathrm{A}=0.45$ (95\% confidence interval, $0.30-0.56$ ). An area under the curve value of $\leq 0.5$ (diagonal line) indicates that the outcome measure was not responsive. $\mathrm{m}$, mid-point.

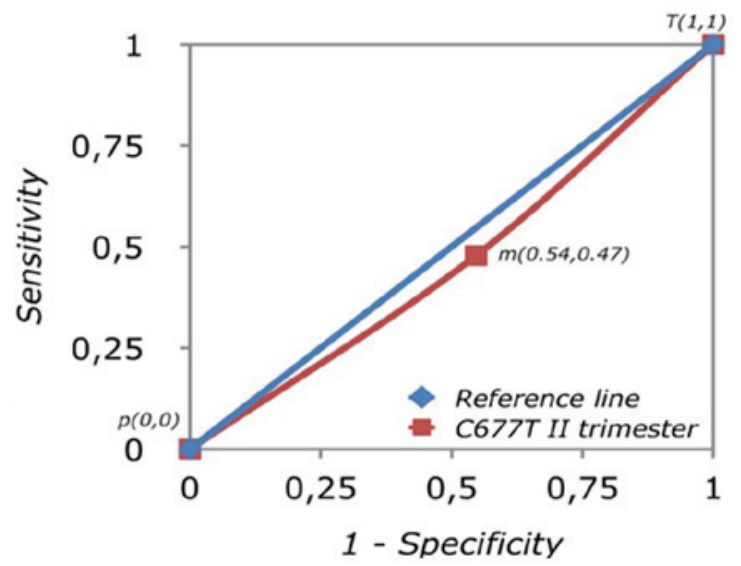

Figure 2. Receiver operating characteristic curve for C677T genotype in the second trimester of pregnancy. The point $\mathrm{m}$ with coordinates $(0.54,0.47)$ together with the coordinate points $(0,0),(1,1)$ and $(1,0)$ defines a quadrilateral. The latter area was given by: $\mathrm{A}=1 / 2$ specificity+ $1 / 2$ sensitivity; $\mathrm{A}=0.46$ (95\% confidence interval, 0.37-0.67). An area under the curve value of $\leq 0.5$ (diagonal line) indicates that the outcome measure was not responsive. $\mathrm{m}$, mid-point.

Groups A and B were further divided into two subgroups based on gestational age [first trimester ( $\leq 13$ weeks of gestation) and second trimester (from 17 weeks and 4 days to 26 weeks and 2 days of gestation)]. Thus, the 380 women in group A were partitioned as those who had a miscarriage during the first trimester of pregnancy (343/380) and those who had a miscarriage in the second trimester of pregnancy (37/380). The 387 women in group B (control group) were partitioned as those subjected to molecular testing in the first trimester of pregnancy (244/387) and those subjected to molecular testing in the second trimester of pregnancy (143/387).

Groups A and B were subjected to preconception prophylaxis (at least one month prior to conception) with oral administration of $400 \mathrm{mcg}(6 \mathrm{~S})-5$-methyltetrahydrofolic acid glucosamine salt, $1.75 \mathrm{~g}$ myo-inositol, $250 \mathrm{mg}$ 


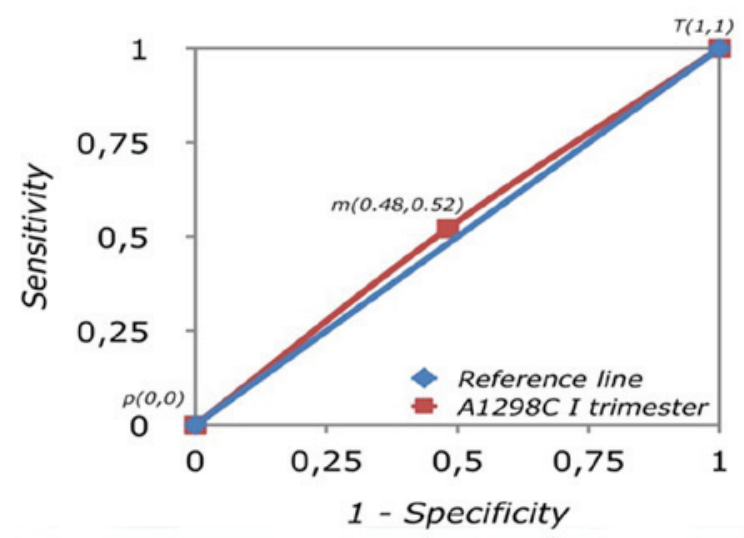

Figure 3. Receiver operating characteristic curve for A1298C genotype in the first trimester of pregnancy. The point $\mathrm{m}$ with coordinates $(0.48,0.52)$ together with the coordinate points $(0,0),(1,1)$ and $(1,0)$ defines a quadrilateral. The latter area was given by: $\mathrm{A}=1 / 2$ specificity $+1 / 2$ sensitivity; $\mathrm{A}=0.51$ (95\% confidence interval, $0.40-0.67$ ). An area under the curve value of $\leq 0.5$ (diagonal line) indicates that the outcome measure was not responsive. $\mathrm{m}$, mid-point.

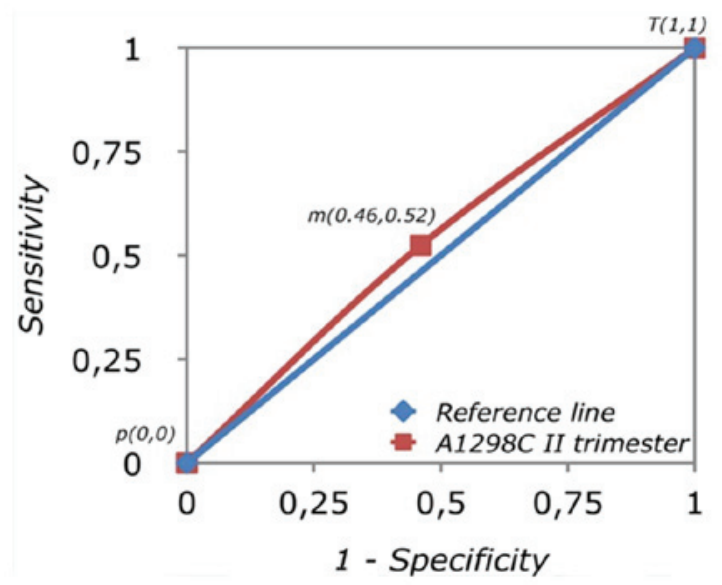

Figure 4. Receiver operating characteristic curve for A1298C genotype in the second trimester of pregnancy. The point $\mathrm{m}$ with coordinates $(0.46,0.52)$ together with the coordinate points $(0,0),(1,1)$ and $(1,0)$ defines a quadrilateral. The latter area was given by: $\mathrm{A}=1 / 2$ specificity $+1 / 2$ sensitivity; $\mathrm{A}=0.52$ (95\% confidence interval, $0.44-0.67$ ). An area under the curve value of $\leq 0.5$ (diagonal line) indicates that the outcome measure was not responsive. $\mathrm{m}$, mid-point.

D-chiro-inositolo, $120 \mathrm{mg}$ vitamin C; $100 \mathrm{mg}$ methylsulfonylmethane and $12.5 \mathrm{mg}$ zinc (Logus Pharma Srl, San Marino, Italy; cat. no. SM22015 47849).

DNA extraction and molecular analysis. For all subjects, molecular testing was performed on venous blood samples (in EDTA-K3) according to the following steps: i) DNA was isolated from $25 \mu \mathrm{l}$ venous blood using a DNA extraction kit obtained from Nuclear Laser Medicine Srl (Settala, Italy; cat. no. AA1001); ii) to study the C677T and A1298C variants of the MTHFR gene, the target genomic area was amplified by polymerase chain reaction (PCR) using two commercial kits provided by Nuclear Laser Medicine Srl [cat. nos. AA901 (C677T) and AA902 (A1298C)]. Following kit protocols, this enabled the detection of single nucleotide polymorphisms using a Rotor-Gene Q MDx (Qiagen Srl, Milan, Italy) using fluorescent oligos annealed to specific DNA target sequences in the gene of interest, and the PCR products were quantified using the Fluorescent Resonance Energy Transfer method (10); iii) the variants of the target sequences were measured from melting curves.

Statistical analysis. The results were compared through receiver operating characteristic (ROC) curve using JMP software version 2 (https://www.jmp.com/it_it/home.html). The ROC curve permits assessment of the accuracy of a diagnostic test. Analysis of a ROC curve is based on the evaluation of the area under the curve (AUC): This expresses the probability that the parameter of an individual randomly selected from the test population is higher than the parameter of an individual randomly selected from the control sample. In reality, it considers appropriate tests with an AUC $\geq 80 \%$. The statistical indicators used to define this graph are specificity (Sp) and sensitivity (Se). The $\mathrm{Sp}$ of a diagnostic test indicates the ability of the test to correctly separate, in the studied population, healthy individuals from individuals carrying the medical condition of interest (11).

\section{Results and Discussion}

MTHFR polymorphism genotypes. Of the 380 pregnant women in group A, 86 heterozygotes and 74 homozygotes for C677T were identified, while 54 heterozygotes and 32 homozygotes for A1298C were identified. A total of 103 were compound heterozygotes, and31 were devoid of mutation.

Of the 387 pregnant women in group B, 100 heterozygotes and 90 homozygotes for C677T were identified. For the A1298C variant, 35 were heterozygous and 32 were homozygous. A total of 110 subjects were compound heterozygotes and 20 lacked mutation.

Comparison of polymorphism genotypes in the first and second trimesters of pregnancy. C677T and A1298C genotype data were compared between the women in groups A and B in their first trimester of pregnancy. Additionally, C677T and A1289C genotype data were compared between the women in groups $\mathrm{A}$ and $\mathrm{B}$ in their second trimester of pregnancy. The results were compared through ROC curve and Z-score statistics.

On ROC curve analysis of all pregnant women in their first trimester of pregnancy based on the presence of C677T in MTHFR, an AUC of 0.45 area units (au) with $95 \%$ confidence interval (CI) 0.30-0.56 was derived (Fig. 1). From a statistical point of view, the area under the reference line is equal to $0.5 \mathrm{au}$ : This value is associated with a diagnostic test without discriminatory qualities, and thus is null. From this data, it may be inferred that there was no association between MTHFR C677T and RPL; therefore, the C677T variant in the considered sample was not useful regarding diagnostic value. The probability of identifying an individual carrying the C677T mutation in the analyzed population was random. These findings were confirmed by the same analysis (C677T vs. WT) performed in the pregnant women in their second trimester of pregnancy. The AUC was 0.46 au with $95 \% \mathrm{CI}$ 0.37-0.67 (Fig. 2).

Analysis of the A1298C variant in MTHFR was subsequently performed. The molecular screening of all women 
in their first trimester obtained an AUC of 0.51 au $(95 \% \mathrm{CI}$ : 0.40-0.67; Fig. 3). In this case, the results indicated that this screening test was not useful. The negative diagnostic application of this molecular screening of A1298C was confirmed by the same analysis in the women in their second trimester: The AUC was 0.52 au (95\% CI: 0.44-0.67; Fig. 4), indicating the random effect that an individual carrying this mutation belonged to the study population rather than the control. This indicated that there was no association between the A1298C variant and RLP risk.

In conclusion, based on the present findings, to carry the C677T and A1298C variants of the MTHFR gene does not appear to influence predisposition to miscarriage in the first or second trimester of pregnancy. Thus, it may be unadvantageous to analyze them for diagnostic aims. However, daily intake of folic acid remains an important therapeutic practice for pregnant women in order to reduce the risk of congenital defects among other complications (12-14). Regarding MTHFR and RPL risk, interaction with other genetic variants has also been speculated (15) and requires investigation.

\section{Acknowledgements}

The authors are thankful to the 'Association Gian Franco Lupo' (ONLUS: a non-profit organization of social utility) for their support.

\section{References}

1. Yang Y, Luo Y, Yuan J, Tang Y, Xiong L, Xu M, Rao X and Liu H: Association between maternal, fetal and paternal MTHFR gene C677T and A1298C polymorphisms and risk of recurrent pregnancy loss: A comprehensive evaluation. Arch Gynecol Obstet 293: 1197-1211, 2016.

2. Levin BL and Varga E: MTHFR: Addressing Genetic Counseling Dilemmas Using Evidence-Based Literature. J Genet Couns 25: 901-911, 2016.

3. Park WC and Chang JH: Clinical Implications of Methylenetetrahydrofolate Reductase Mutations and Plasma Homocysteine Levels in Patients with Thromboembolic Occlusion. Vasc Spec Int 30: 113-119, 2014.
4. Baum L, Wong KS, Ng HK, Tomlinson B, Rainer TH, Chan DK, Thomas GN, Chen X, Poon P, Cheung WS, et al: Methylenetetrahydrofolate reductase gene A222V polymorphism and risk of ischemic stroke. Clin Chem Lab Med 42: 1370-1376, 2004.

5. Sibani S, Leclerc D, Weisberg IS, O'Ferrall E, Watkins D, Artigas C, Rosenblatt DS and Rozen R: Characterization of mutations in severe methylenetetrahydrofolate reductase deficiency reveals an FAD-responsive mutation. Hum Mutat 21: 509-520, 2003.

6. Botto N, Andreassi MG, Manfredi S, Masetti S, Cocci F, Colombo MG, Storti S, Rizza A and Biagini A: Genetic polymorphisms in folate and homocysteine metabolism as risk factors for DNA damage. Eur J Hum Genet 11: 671-678, 2003.

7. Friso $S$ and Choi SW: Gene-nutrient interactions and DNA methylation. J Nutr 132: 2382S-2387S, 2002.

8. Dell'edera D, Tinelli A, Milazzo GN, Malvasi A, Domenico C, Pacella E, Pierluigi C, Giuseppe T, Marcello G, Francesco L, et al: Effect of multivitamins on plasma homocysteine in patients with the 5,10 methylenetetrahydrofolate reductase C677T homozygous state. Mol Med Rep 8: 609-612, 2013.

9. Martinez-Serra J, Robles J, Nicolàs A, Gutierrez A, Ros T, Amat JC, Alemany R, Vögler O, Abelló A, Noguera A, et al: Fluorescence resonance energy transfer-based real-time polymerase chain reaction method without DNA extraction for the genotyping of F5, F2, F12, MTHFR, and HFE. J Blood Med 5: 99-106, 2014.

10. Pritchett YL and Tamura R: Global benefit-risk assessment in designing clinical trials and some statistical considerations of the method. Pharm Stat 7: 170-178, 2008.

11. Schmidt RJ, Tancredi DJ, Ozonoff S, Hansen RL, Hartiala J, Allayee H, Schmidt LC, Tassone F and Hertz-Picciotto I: Maternal periconceptional folic acid intake and risk of autism spectrum disorders and developmental delay in the CHARGE (CHildhood Autism Risks from Genetics and Environment) case-control study. Am J Clin Nutr 96: 80-89, 2012.

12. Parker SE, Yazdy MM, Tinker SC, Mitchell AA and Werler MM: The impact of folic acid intake on the association among diabetes mellitus, obesity, and spina bifida. Am J Obstet Gynecol 209: 239.e1-239.e8, 2013.

13. Friberg AK and Jørgensen FS: Periconceptional folic acid prophylaxis and neural tube defects. Ugeskr Laeger 177: 1595-1598, 2015 (In Danish)

14. Greene ND, Leung KY, Gay V, Burren K, Mills K, Chitty LS and Copp AJ: Inositol for prevention of neural tube defects: A pilot randomised controlled trial - CORRIGENDUM. Br J Nutr 115: 1697,2016

15. Settin A, Elshazli R, Salama A and ElBaz R: Methylenetetrahydrofolate reductase gene polymorphisms in Egyptian women with unexplained recurrent pregnancy loss. Genet Test Mol Biomarkers 15: 887-892, 2011. 